\title{
High-Frequency Fiber Bragg Grating Sensing Interrogation System Using Sagnac-Loop-Based Microwave Photonic Filtering
}

\author{
Hongyan Fu, Wei Zhang, Chengbo Mou, Xuewen Shu, Lin Zhang, Sailing He, Senior Member, IEEE, and \\ Ian Bennion
}

\begin{abstract}
A novel high-frequency fiber Bragg grating (FBG) sensing interrogation system by using fiber Sagnac-loop-based microwave photonic filtering is proposed and experimentally demonstrated. By adopting the microwave photonic filtering, the wavelength shift of sensing FBG can be converted into amplitude variation of the modulated electronic radio-frequency (RF) signal. In the experiment, the strain applied onto the sensing FBG has been demodulated by measuring the intensity of the recovered RF signal, and by modulating the RF signal with different frequencies, different interrogation sensitivities can be achieved.
\end{abstract}

Index Terms-Chirped fiber Bragg grating (CFBG), microwave photonics, Sagnac loop, strain sensor.

\section{INTRODUCTION}

$\mathbf{F}$ IBER Bragg grating (FBG) sensors have been studied extensively for more than two decades, due to their advantages such as compactness, light weight, immunity to electromagnetic interference, and ease to be embedded and multiplexed. FBG sensors can be applied to measure a wide range of parameters including strain, temperature, surrounding medium refractive index, loading, and bending [1], [2]. The operation of FBG sensors is normally based on the dependence of their reflection or transmission spectrum on the measurands. Since most FBG-based sensors are wavelength-encoded, practical interrogation system requires the detection of the wavelength shift and their conversion to intensity variation.

Many interrogation schemes for FBG sensors have been reported [3], [4]. Most of these interrogation techniques can only handle static or low-frequency wavelength variation introduced by FBGs. For some applications such as acoustic emission detection, the typical values for ultrasonic signal frequencies used

Manuscript received November 10, 2008; revised December 24, 2008. First published February 03, 2009; current version published March 27, 2009. This work was supported by the Chinese Scholarship Council.

H. Fu is with the Centre for Optical and Electromagnetic Research, State Key Laboratory of Modern Optical Instrumentation, Zhejiang University, Zijingang Campus, Hangzhou 310058, China and also with the Photonics Research Group, Aston University, Birmingham, B4 7ET, U.K. (e-mail: fuhongyan@coer.zju. edu.cn).

W. Zhang, C. Mou, X. Shu, L. Zhang, and I. Bennion are with the Photonics Research Group, Aston University, Birmingham, B4 7ET, U.K. (e-mail: w.zhang@aston.ac.uk; mouc@aston.ac.uk; x.shu@aston.ac.uk; 1.zhang@aston.ac.uk; i.bennion@aston.ac.uk).

S. He is with the Centre for Optical and Electromagnetic Research, State Key Laboratory of Modern Optical Instrumentation, Zhejiang University, Zijingang Campus, Hangzhou 310058, China. (e-mail: sailing@ zju.edu.cn).

Color versions of one or more of the figures in this letter are available online at http://ieeexplore.ieee.org.

Digital Object Identifier 10.1109/LPT.2009.2014077

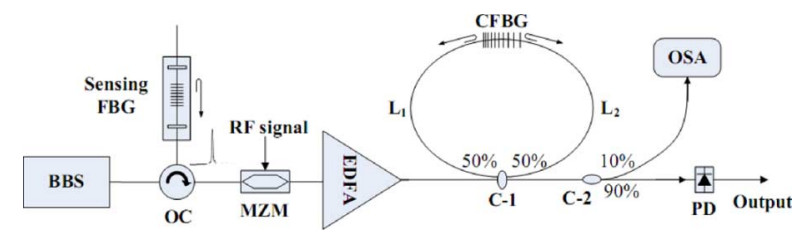

Fig. 1. Setup for the proposed interrogation system. BBS: broadband optical source. OC: optical circulator. MZM: Mach-Zehnder modulator. C-1: coupler 1. C-2: coupler 2.

in the nondestructive testing technology range from $100 \mathrm{kHz}$ up to several megahertz. There are so few cost-effective interrogation techniques available for high-frequency wavelength demodulation. Some reported techniques using fiber-optic Mach-Zehnder interferometers [5], [6] claimed capable of the high-frequency wavelength demodulation, they all suffer from the problems associated with fiber-optic interferometer such as polarization fading and reliability in crucial environment. With the development of photonic and mobile networks technology, the realization of microwave photonic signal processing has become more and more convenient and cost-effective [7]. This technique has started attracting research attention in the applications of interrogating fiber gating sensors [8], [9] due to its capability in handling fast signal processing speed.

Incorporating a linear chirped fiber Bragg grating (LCFBG), the loop generates a microwave photonic notch response and converts the wavelength shift of the sensing FBG into the intensity change at the modulated radio frequencies (RFs). In this letter, this microwave photonic filtering-based sensing interrogation system is studied comprehensively in the experiment. This interrogation scheme is simple in configuration and can handle high-frequency wavelength variation, thus having potential in remote, multiplexed, and wireless sensing systems with high-frequency wavelength modulation.

\section{OPERATION PRINCIPLE}

The basic principle of the interrogation scheme by using Sagnac-loop-based microwave photonic filtering structure has been proposed in [10]. Fig. 1 shows the arrangement of the proposed Sagnac-loop-based FBG sensing interrogation scheme. The output of this structure is a typical response of microwave photonic notch filter, whose frequency response can be expressed as

$$
P_{e}=P_{0}\left[1+m \cos \left(\frac{\Delta \phi}{2}\right) \sin \left(2 \pi f t+\phi_{0}\right)\right]
$$


where $P_{0}$ is the optical power reflected by the sensing FBG, $m$ is the modulation index of the MZM, $f$ is the modulation frequency, and $\phi_{0}$ is the phase of the output electrical signal from the photodiode (PD). $\Delta \phi=4 \pi f \cdot n_{\mathrm{eff}} \cdot\left(L_{1}-L_{2}+2 z-l\right) / c$ is the phase difference of the modulated signal experienced in two arms of the Sagnac-loop, and $L_{1}$ and $L_{2}$ are the lengths of two arms; $n_{\mathrm{eff}}, c$, and $l$ are the refractive index of the fiber, speed of light in vacuum, and the length of LCFBG, respectively; $z$ is the position in the LCFBG where the light is reflected, and decided by the Bragg wavelength of the sensing FBG.

For this type of microwave photonic filtering, the peak frequency where the filter response reaches the maximum can be defined as

$$
f_{\text {peak }}=N \cdot \mathrm{FSR}=\frac{N}{\tau}
$$

where $N=0,1,2, \ldots, \mathrm{FSR}$ and $\tau$ are the free-spectral range of the notch filter and time difference between the two arms of the Sagnac-loop, respectively. Obviously as the strain changes the reflection wavelength, thus changing the path difference between the two arms, the peak frequency varies with the applied strain to the sensing grating. If we track one of the peak frequencies, it will be a function of the wavelength of sensing grating, thus the applied strain.

The interesting feature of this interrogation scheme is that the rate of this peak frequency change against the applied strain is proportional to the frequency itself, i.e., the higher this peak frequency is, the larger change will occur, according to (2). The interrogation responsive factor against the applied strain by tracking the peak frequency can be defined as (3), which shows that if we choose the $N$ th peak to track, its responsive factor will be $N$ times of that of the first peak frequency

$$
\frac{d f_{\text {peak }}}{d \varepsilon} \propto-N \lambda_{B} \frac{4 n_{\text {eff }}}{c \tau^{2} C}
$$

where $\lambda_{B}$ is the Bragg wavelength of the sensing grating, $C$ is the grating chirp rate.

Instead of tracking the change of the peak frequency, monitoring the amplitude change at a fixed modulation frequency is more practical in terms of realization and cost of signal processing electronics. From (1), the measurement responsive factor can be deduced as

$$
\left.\frac{d P_{e}}{d \varepsilon}\right|_{f} \propto-m \sin \left(\frac{\Delta \phi}{2}\right) \frac{4 \pi f n_{\mathrm{eff}}}{c C} .
$$

Again, the responsive factor is proportional to the RF frequency.

\section{EXPERIMENT SETUP AND RESULTS}

In the experiment, the Bragg wavelength of the sensing FBG is originally located at $1553 \mathrm{~nm}$. The length of the LCFBG is around $10 \mathrm{~cm}$, and its reflection, bandwidth, and time delay are around $10 \mathrm{~dB}, 7 \mathrm{~nm}$, and $1000 \mathrm{ps}$, respectively. The reflection spectrum and the time delay of the LCFBG are shown in Fig. 2. After the LCFBG Sagnac-loop, $90 \%$ of the light from C-2 is sent to the PD (New Focus Inc.), while the other 10\% of light is monitored by the optical spectrum analyzer [(OSA) HP86142A]. The frequency response of the detected signal can be measured by the lightwave component analyzer [(LCA) HP8703A]. The output signal modulated at a fixed frequency is monitored by

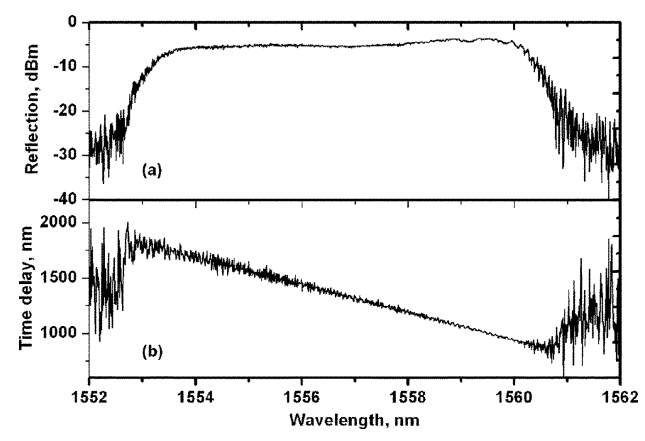

Fig. 2. (a) Measured reflection spectrum and (b) time delay of the LCFBG.

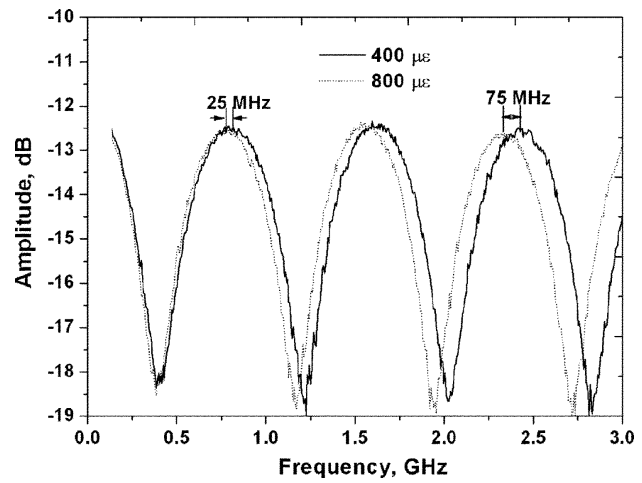

Fig. 3. Measured frequency responses when different strains are applied: $400-\mu$ strain (solid line) and $800-\mu$ strain (dotted line).

an electronic spectrum analyzer [(ESA) HP8562A], which has a $10-\mathrm{dB}$ attenuation at the input.

Fig. 3 shows the measured microwave photonic filtering frequency responses up to $3 \mathrm{GHz}$ for two strains -400 and $800 \mu \varepsilon$-applied to the sensing FBG. From Fig. 3, we can see that the first peak frequency $(N=1)$ appears at $\sim 567 \mathrm{MHz}$ and the frequency change over $400 \mu \varepsilon$ is about $-25 \mathrm{MHz}$; the third peak $(N=3)$ appears at $\sim 1.7 \mathrm{GHz}$ and the corresponding frequency change is about $-75 \mathrm{MHz}$, which is exactly three times of that induced for the first peak. The fact that higher order peak introduces larger change offers a great flexibility in setting the responsive factor according to different applications.

The measured responses of the second peak frequency and the sensing FBG wavelength against the applied strain are shown in Fig. 4(a) where the peak frequency decreases with increased strain. Fig. 4(b) shows the relationship between the recovered RF signal intensity and the wavelength of the sensing FBG at different modulation RF frequencies. From the results, one can see that, when the optical signal is modulated at $473 \mathrm{MHz}$, the intensity of the recovered signal increases as the applied strain increases, that is because the modulation frequency is located in the rising slope of the filtering response. When the optical signal is modulated $992 \mathrm{MHz}$ and $1.22 \mathrm{GHz}$, respectively, the intensity of recovered signal decreases with the increased strain as the modulation frequency is located in the falling slope of the filtering response. Also we can see from Fig. 4(b) that this measurement responsive factor is related to the modulation frequency which agrees with (4). We can see from the experimental results that the responsive factor of the interrogation system at $1.22 \mathrm{GHz}, 992 \mathrm{MHz}$, and $473 \mathrm{MHz}$ are $-524,-340$, and 

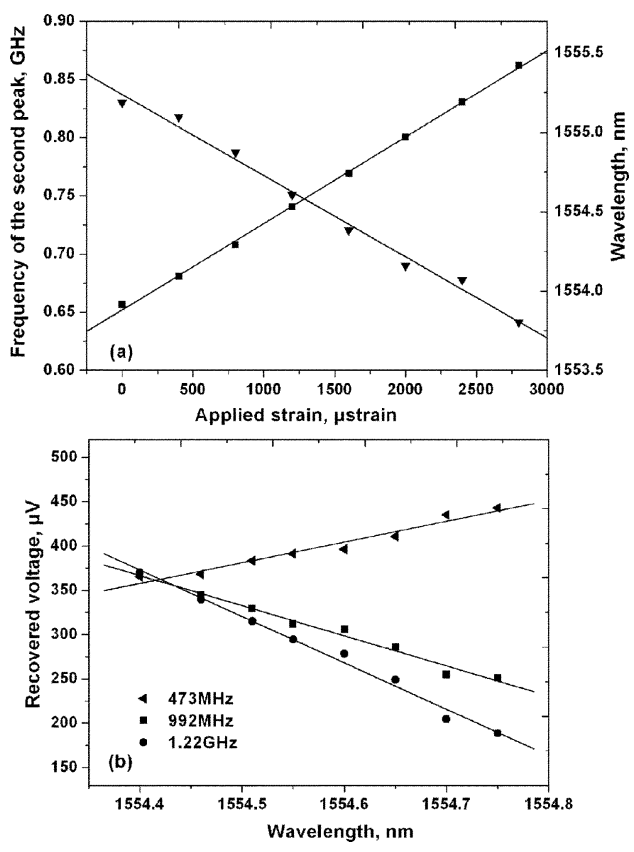

Fig. 4. (a) Relationship of the frequency of second peak (triangles) and the wavelength of sensing FBG (squares) with the applied strain. (b) Relationship between recovered RF intensity and the wavelength of sensing FBG at different modulation frequencies (473 MHz: triangles; $995 \mathrm{MHz}$ : squares; $1.22 \mathrm{GHz}$ : circles).



Fig. 5. (a) Measured waveform of the original 20-Hz signal sent to the PZT; time responses for a $20-\mathrm{Hz}$ alternating strain when the light beam is modulated at (b) 8.3 and (c) $3.7 \mathrm{GHz}$.

$234 \mu \mathrm{V} / \mathrm{nm}$, respectively, and for these selected modulation frequencies, higher frequency has higher interrogation responsive factor.

A dynamic strain was then applied to the sensing FBG by mounting it on a piezo stage driven by a function generator. Fig. 5 shows the waveform of original $20-\mathrm{Hz}$ signal applied to PZT, the time responses of detected optical signal at 3.7 and $8.3 \mathrm{GHz}$, measured by the LCA while a $20-\mathrm{Hz}$ alternating strain was applied to the sensing FBG. One can see that the response of higher frequency signal is larger than that of lower frequency, which means that for the proposed interrogation system, higher modulation frequency offers higher interrogation responsive factor.

The interrogation of higher frequency wavelength change can be expected by using the same technique. However, the performance of this proposed interrogation system is greatly af- fected by the LCFBG in use, whose ripple in time delay response limits the minimum detectable wavelength change, since the time delay of LCFBG plays an important part in the time difference between two arms of the Sagnac-loop, which according to (1) will have influence on the recovered intensity. From (3) and (4), the responsive factor and minimum detectable strain level can also be affected by the chirp rate of the LCFBG so the detection capability can be tailored by choosing LCFBG with different chirp rates. The system performance can be further improved by improving the quality of the LCFBG and using other dispersive devices. It should be pointed out that according to (4), the responsive factor is maximum and monotonically proportional to modulation frequency only when $\Delta \phi / 2=\pi / 2$; it reaches minimum when $\Delta \phi / 2=0$. Therefore, the modulation frequency should be set carefully to ensure the optimum responsivity. This explains why in this work the responses obtained at different modulation frequencies do not vary at the same rate as modulation frequencies.

\section{CONCLUSION}

In this letter, an FBG sensing interrogation system using Sagnac-loop-based microwave photonic filtering has been proposed and demonstrated. By using a fiber Sagnac-loop incorporating an LCFBG, the wavelength shift of the sensing FBG can be converted into the intensity change at RF modulation frequency. The measurement responsive factor of this proposed technique is proportional to the modulation frequency used. It offers a great advantage in the situation where a high measurement responsive factor is a priority and flexibility for static and dynamic measurement is required. This sensing interrogation system will benefit those applications in remote, wireless, and multiplexed sensing systems with the requirement for high-frequency wavelength demodulation.

\section{REFERENCES}

[1] T. Erdogan, "Fiber grating spectra," J. Lightw. Technol., vol. 15, no. 8, pp. 1277-1294, Aug. 1997.

[2] Y.-J. Rao, "In-fibre Bragg grating sensors," Meas. Sci. Technol., vol. 8, pp. 335-375, 1997.

[3] S. Kim, S. Kim, J. Kwon, and B. Lee, "Fiber Bragg grating strain sensor demodulator using a chirped fiber grating," IEEE Photon. Technol. Lett., vol. 13, no. 8, pp. 839-1841, Aug. 2001.

[4] D. Zhao, X. Shu, Y. Lai, L. Zhang, and I. Bennion, "Fiber Bragg grating sensor interrogation using chirped fiber grating-based Sagnac loop," IEEE Sensors J., vol. 3, no. 6, pp. 734-737, Dec. 2003.

[5] A. D. Kersey, T. A. Berkoff, and W. W. Morey, "High-resolution fibregrating based strain sensor with interferometric wavelength-shift detection," Electron. Lett., vol. 28, no. 3, pp. 236-238, 1992.

[6] N. E. Fisher, J. Surowiec, D. J. Webb, D. A. Jackson, L. R. Gavrilov, J. W. Hand, L. Zhang, and I. Bennion, "In-fibre Bragg gratings for ultrasonic medical applications," Meas. Sci. Technol., vol. 8, pp. 1050-1054, 1997.

[7] J. Capmany, B. Ortega, and D. Pastor, "A tutorial on microwave photonic filters," J. Lightw. Technol., vol. 24, no. 1, pp. 201-229, Jan. 2006.

[8] B. Noharet, M. Turpin, J. Chazelas, P. Bonniau, D. Walsh, W. C. Mishie, and B. Culshaw, "Microwave subcarrier optical fiber strain sensor," in 2nd Eur. Conf. Smart Structures and Materials, Glasgow, U.K., 1994, Session 6.

[9] G. Gagliardi, M. Salza, P. Ferraro, and P. De Natale, "Fiber Bragggrating strain sensor interrogation using laser radio-frequency modulation," Opt. Express, vol. 13, pp. 2377-2384, 2005.

[10] H. Fu, W. Zhang, C. Mou, X. Shu, L. Zhang, S. He, and I. Bennion, "High frequency sensing interrogation system using fiber Bragg grating based microwave photonic filtering," in OFC 2009, San Diego, CA, Paper JWA9. 